\title{
Marked elevation of B-type natriuretic peptide in patients with heart failure and preserved ejection fraction
}

\author{
Samuel Tate ${ }^{\mathrm{a}}$, Andrea Griem ${ }^{\mathrm{a}}$, Blythe Durbin-Johnson ${ }^{\mathrm{b}}$, Clifton Watt ${ }^{\mathrm{a}}$, Saul Schaefer ${ }^{\mathrm{a}, \mathrm{c},}$ 凶 \\ ${ }^{a}$ Department of Internal Medicine, Division of Cardiovascular Medicine, University of California Davis, Davis, CA, USA; \\ ${ }^{b}$ Department of Public Health Sciences, Division of Biostatistics, University of California Davis, Davis, CA, USA; \\ ${ }^{c}$ Cardiology Section, Department of Veteran Affairs, Northern California Health Care System, Mather, CA, USA. \\ Received 06 February 2014, Revised 08 April 2014, Accepted 15 April 2014, Epub 07 June 2014
}

\begin{abstract}
Marked elevations of B-type natriuretic peptide (BNP) are not generally seen in patients with heart failure and preserved ejection fraction (HFpEF). The objective of this study was to examine the clinical and laboratory characteristics of a large cohort of patients with HFpEF and markedly elevated BNP. A retrospective examination of 421 inpatients at a university hospital admitted with a diagnosis of HFpEF was performed. Clinical and echocardiographic data in 4 groups of patients with levels of BNP $\leq 100 \mathrm{pg} / \mathrm{mL}, 100-400 \mathrm{pg} / \mathrm{mL}, 400-1,000 \mathrm{pg} / \mathrm{mL}$ and $>1,000 \mathrm{pg} / \mathrm{mL}$ were compared. Patients with HFpEF and BNP $>1,000 \mathrm{pg} / \mathrm{mL}$ ( $28 \%$ of the population) were characterized by impaired renal function and greater use of anti-hypertensive medications. A subset of these patients with $\mathrm{BNP}>1,000 \mathrm{pg} / \mathrm{mL}$ had normal renal function $(21 \%)$ and were significantly older, more frequently female, and tended to have lower ejection fractions. Conversely, patients with $\mathrm{HFpEF}$ and $\mathrm{BNP} \leq 100 \mathrm{pg} / \mathrm{mL}$ were younger and had preserved renal function. BNP was inversely related to the likelihood of subsequent admission for heart failure, but not to myocardial infarction or death. In conclusion: BNP $>1,000 \mathrm{pg} / \mathrm{mL}$ is seen in almost $1 / 3$ of patients hospitalized with HFpEF. This elevation of BNP often reflects impaired renal function, but can also be seen in patients with preserved renal function but relatively impaired systolic function.
\end{abstract}

Keywords: B-type natriuretic peptide, diastolic heart failure, chronic kidney disease

\section{INTRODUCTION}

Heart failure with preserved ejection fraction (HFpEF) is becoming a more common diagnosis as the prevalence of patients with hypertension, diabetes, chronic kidney disease (CKD) and advancing age increases. HFpEF is now the cause of clinical heart failure in approximately $50 \%$ of patients, is a frequent cause of hospitalization, and is associated with significant morbidity and mortality ${ }^{[1]}$. The diagnosis of HFpEF depends on the clinical diagnosis of heart failure in the setting of preserved ejection fraction, usually with an ejection fraction $>45 \%{ }^{[1]}$. In addition to clin-

\footnotetext{
${ }^{\square}$ Corresponding author: Saul Schaefer, M.D., University of California, One Shields Avenue, Davis, CA 95616, USA. Tel/Fax: (530) 752-0718/

ical assessment, the severity of heart failure is often assessed by measuring B-type natriuretic peptide (BNP), a peptide hormone released by cardiomyocytes in response to increased wall stress ${ }^{[2]}$. In contrast to heart failure with impaired systolic function ( $\mathrm{EF} \leq 45 \%)$ where serum levels of BNP are often $>1,000 \mathrm{pg} / \mathrm{mL}$ in patients with severe dysfunction ${ }^{[3]}$, the levels of BNP in HFpEF tend to be lower, with mean values in the literature reported in the range of $400-500 \mathrm{pg} / \mathrm{mL}^{[2]}$. However, BNP values $>1,000$ can now be seen in $\mathrm{HFpEF}^{[4]}$ and may denote a worse prognosis. In order to characterize and identify these patients, the purpose of this study was to examine the clinical and echocardio-

(530) 752-3264, E-mail: sschaefer@ucdavis.edu. The authors reported no conflict of interests. 
graphic characteristics of patients with markedly elevated BNP.

\section{PATIENTS AND METHODS}

\section{Patients and patient selection}

This study was approved by the Institutional Review Board of the University of California Davis.

Patients admitted to the University of California Davis Medical Center between 7/1/2010 and 6/30/2011 with a diagnosis of HFpEF (ICD9 code 428) were examined. The diagnosis of HFpEF was made by the hospital attending physician and was based on clinical signs and symptoms of heart failure with imaging evidence of preserved left ventricular ejection fraction $(>45 \%)$ by echocardiography. Demographic, clinical, and echocardiographic data were obtained from chart review of the electronic medical record and the echocardiographic data base. The characteristics of the patients are shown in Table 1. A total of 421 patients met entry criteria, and

Table 1 Patient characteristics by BNP category [mean (SD)]

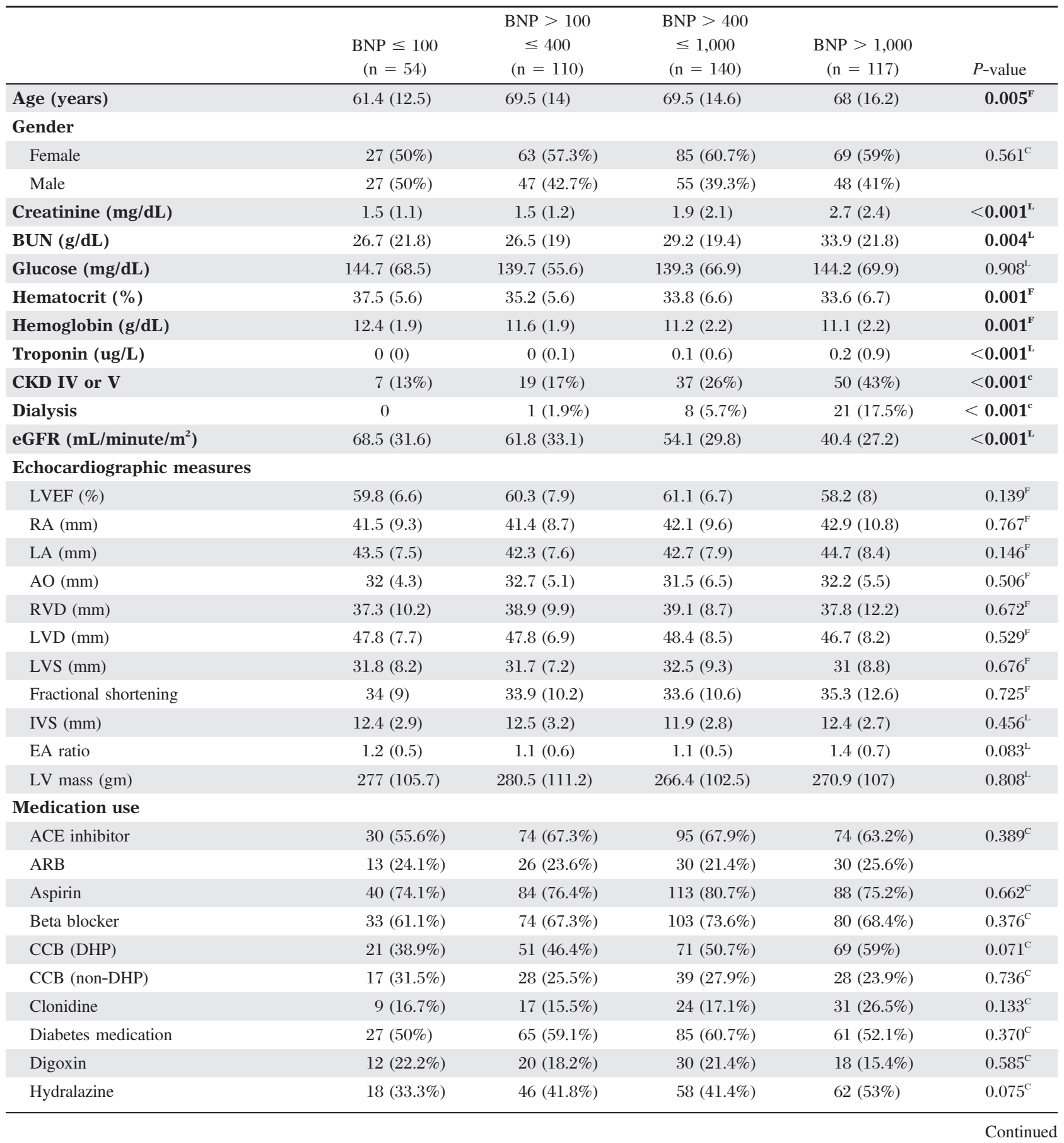


Table 1 Patient characteristics by BNP category [mean (SD)] (continued)

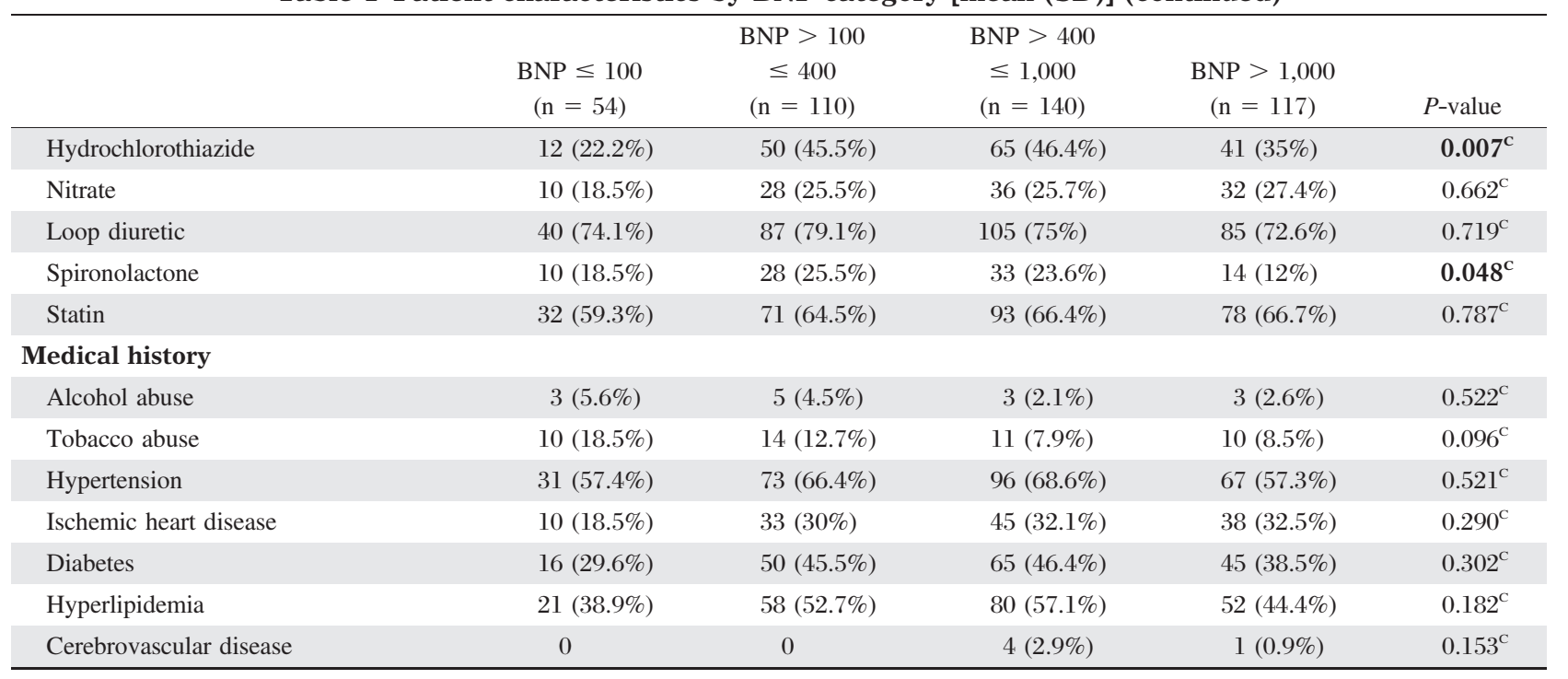

$\mathrm{F}=P$-value for global test of any differences between BNP categories from ANOVA F-test. $\mathrm{L}=P$-value from ANOVA F-test, data log transformed prior to analysis. $\mathrm{C}=P$-value from chi-square test. $\mathrm{K}=P$-value from Kruskal-Wallis test. LVEF: left ventricular ejection fraction; RA: right atrium; LA: left atrium; AO: aorta; RVD: right ventricle; LVD: left ventricle diastole; LVS: left ventricle systole; IVS: interventricular septum; DHP: dihydropyridine.

were analyzed based on the maximum BNP values obtained during the index hospitalization. BNP was measured using an immunoenzymatic assay (AlereInc, Waltham MA). Concurrent echocardiography data were obtained including left ventricular ejection fraction, chamber dimensions, left ventricular mass and E/A ratio. These measurements were derived from the echocardiographic images using American Society of Echocardiography standards ${ }^{[5]}$. Cardiovascular outcomes (death, myocardial infarction, and rehospitalization for heart failure) were recorded for a mean of 2.7 years.

\section{Data analysis}

Patients were grouped based on BNP levels $(\leq 100$, $>100$ and $\leq 400,>400$ and $\leq 1,000$, and $>$ $1,000 \mathrm{pg} / \mathrm{mL}$ ). Continuous patient characteristics were compared between BNP groups using ANOVA, with the data $\log$ transformed where a histogram indicated data skewness. For patient characteristics with a significant ANOVA F-test for the global test of any differences among BNP categories, pairwise comparisons between groups were conducted using the TukeyKramer method. Frequencies for categorical patient characteristics were compared between groups using chi-square tests. In the case of a significant chi-square test, adjusted residuals ${ }^{[6]}$ were examined to determine the nature of the differences between groups. The Kruskal-Wallis test was used to compare median CKD stage between groups. This analysis was repeated in patients with BNP $>1000$, comparing patients with and without a history of CKD (eGFR $>60 \mathrm{~mL} / \mathrm{min} / \mathrm{m}^{2}$ as determined by the MDRD equation ${ }^{[7]}$ ). A multiple linear regression model was used to estimate the joint effects of selected patient characteristics on BNP levels. Variables selected for inclusion in the model were those that differed significantly between BNP groups in univariate analysis. Data are presented as mean $+/$ - standard deviation (SD). A $P$ value $\leq 0.05$ was used to reject the null hypothesis.

\section{RESULTS}

Patient characteristics are shown in Table 1. Patients with a BNP $>1,000 \mathrm{pg} / \mathrm{mL}$ comprised $28 \%$ of inpatients with a clinical diagnosis of HFpEF. Patients with BNP > $1,000 \mathrm{pg} / \mathrm{mL}$, compared to those with a BNP $\leq 100 \mathrm{pg} /$ $\mathrm{mL}$, were characterized by a significantly higher age, higher levels of serum creatinine and BUN, lower eGFR, a higher prevalence of CKD IV or V and use of dialysis, lower hemoglobin, higher peak levels of troponin, and greater use of anti-hypertensive medications such as thiazides and spironolactone. However, when compared to all other patients, only eGFR and troponin was significantly different in the patients with BNP > $1,000 \mathrm{pg} / \mathrm{mL}$. When BNP was plotted as a function of eGFR, there was a significant relationship, $r=0.39$ (Fig. 1). Importantly, variables that were not significantly different between BNP groups included left ventricular size, ejection fraction, or wall thickness, as well as sex, diagnosis of diabetes or ischemic heart disease, and use of beta-blockers.

In order to examine the effect of renal dysfunction and reduced clearance on the serum level of BNP, 


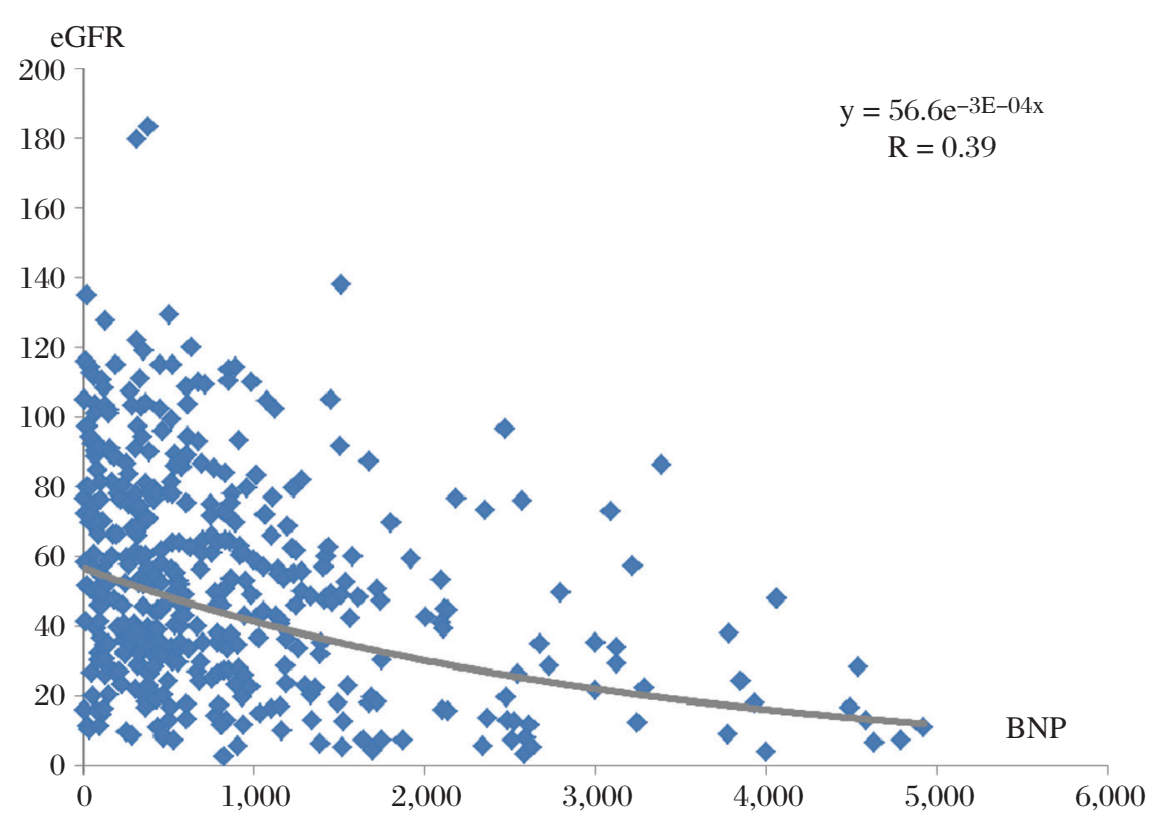

Fig. 1 Relationship of BNP (pg/mL) and renal function (eGFR, $\left.\mathrm{mg} / \mathrm{minute} / \mathrm{m}^{2}\right)$ for the entire cohort of patients. The relationship between these 2 variables was statistically significant, $r=0.39, P \leq 0.05$. Correlation coefficient derived using log transformation of the data.

the characteristics of patients with BNP $>1,000 \mathrm{pg} / \mathrm{mL}$ but without CKD (defined as eGFR $>60 \mathrm{~mL} /$ minute $/ \mathrm{m}^{2}$ ) were examined and compared to those patients with CKD (Table 2). In comparison to patients with CKD, the patients with a BNP $>1,000 \mathrm{pg} / \mathrm{mL}$ but without CKD, comprising $21 \%$ of patients, had marginally lower mean values of BNP $(1,677+/-608$ vs $2,101+/$ $1,063 \mathrm{pg} / \mathrm{mL}), P=0.06$, and a lower use of antihypertensive medications such as calcium channel blockers, clonidine, and hydralazine. Echo parameters also differed significantly between these two groups, with patients without $\mathrm{CKD}$ having a lower $\mathrm{E} / \mathrm{A}$ ratio $(1.0+/-0.6$ vs. $1.4+/-0.7)$, but a greater LV mass $(335.9+/-138.9$ vs. $273.8+/-106.5$ gms, $P<0.05)$. There was a trend toward lower ejection fractions in those patients without CKD (LVEF 55.4 +/- 9.3 vs. $59.0+/-7.7, P=0.08$ ).

Patients with a clinical diagnosis of HFpEF but normal BNP $(\leq 100 \mathrm{pg} / \mathrm{mL})$ comprised $13 \%$ of the study population and were characterized by younger age $(61.4+/-12.5$ years $)$ and preserved renal function (eGFR $68.8+/-31.9 \mathrm{~mL} /$ minute $/ \mathrm{m}^{2}$ ), although 13 of $54(24 \%)$ patients in this group had creatinine levels greater than the upper limit of normal $(1.28 \mathrm{gm} / \mathrm{dL})$, with values as high as $5.87 \mathrm{mg} / \mathrm{dL}$.

The level of BNP in the index hospitalization did not predict subsequent death or hospitalization for a myocardial infarction. However, BNP levels were lower in patients subsequently hospitalized for heart failure during the follow-up period $(631.2+/-647.7 \mathrm{vs}$ $907.2+/-956.8 \mathrm{pg} / \mathrm{mL}, P=0.04)$.

\section{DISCUSSION}

The role of B-type natriuretic peptide in heart failure

B-type natriuretic peptide (BNP) is a peptide hormone released in response to increased ventricular wall stress, and is often used to diagnose heart failure due to systolic dysfunction $(\mathrm{HFrEF})^{[7]}$. In patients with heart failure with preserved ejection fraction (HFpEF), elevations in BNP have been reported to correlate with left ventricular end-diastolic wall stress ${ }^{[8]}$, age, and abnormal diastolic function (assessed by left atrial volume index), and inversely with LV ejection fraction $^{[9]}$. Median values of BNP in patients with HFpEF are generally lower than those with reduced ejection fraction, with median values of $413^{[10]}$ and $445 \mathrm{pg} / \mathrm{mL}^{[2]}$ previously being reported in these patients. Thus, BNP values $>1,000 \mathrm{pg} / \mathrm{mL}$ are unusual and may identify a different cohort of patients at different risk for further cardiovascular events ${ }^{[4]}$.

\section{Clinical predictors of BNP in HFpEF}

In the current study, the prevalence of BNP $>$ $1,000 \mathrm{pg} / \mathrm{mL}$ was $28 \%(117 / 421)$ and $\mathrm{BNP}>$ $2,000 \mathrm{pg} / \mathrm{mL}$ was $11 \%(45 / 421)$. Patients with a BNP $>1,000 \mathrm{pg} / \mathrm{mL}$, when compared to those with a normal BNP $(\leq 100 \mathrm{pg} / \mathrm{mL})$, were characterized by significantly higher age, worse renal function, anemia, elevated troponins, and use of anti-hypertensive agents. Important variables that were not associated with a markedly ele- 
Table 2 Patient characteristics by CKD - patients with BNP $>1,000(\mathrm{pg} / \mathrm{mL})$

\begin{tabular}{|c|c|c|c|}
\hline & $\begin{array}{l}\text { No CKD } \\
(\mathrm{n}=24)\end{array}$ & $\begin{array}{c}\text { CKD } \\
(\mathrm{n}=93)\end{array}$ & P-Value \\
\hline Age (years) & $70.2(13.5)$ & $67.7(16.3)$ & $0.493^{\mathrm{L}}$ \\
\hline \multicolumn{4}{|l|}{ Gender } \\
\hline Female & $13(54.2 \%)$ & $54(58.1 \%)$ & $0.731^{\mathrm{C}}$ \\
\hline Male & $11(45.8 \%)$ & $39(41.9 \%)$ & \\
\hline BNP & 1677 (608) & $2101(1063)$ & $0.064^{\mathrm{L}}$ \\
\hline eGFR & $81.9(17.6)$ & $29.9(17.5)$ & $<0.001^{\mathrm{L}}$ \\
\hline Creatinine & $0.9(0.16)$ & $3.2(2.5)$ & $<0.001^{\mathrm{L}}$ \\
\hline BUN & $15.5(6.7)$ & $38.8(22.6)$ & $<0.001^{\mathrm{L}}$ \\
\hline Glucose & $139.8(46.8)$ & $147.7(79)$ & $0.857^{\mathrm{L}}$ \\
\hline Hematocrit & $35.4(5)$ & $32.7(7.3)$ & $0.043^{F}$ \\
\hline Hemoglobin & $11.7(1.8)$ & $10.7(2.4)$ & $0.025^{\mathrm{F}}$ \\
\hline Troponin & $0.4(1.5)$ & $0.1(0.2)$ & $0.697^{\mathrm{L}}$ \\
\hline \multicolumn{4}{|c|}{ Echocardiographic measures } \\
\hline LVEF & $55.4(9.3)$ & $59.0(7.7)$ & $0.077^{\mathrm{F}}$ \\
\hline RA & $43.5(12.3)$ & $42.6(10.2)$ & $0.712^{\mathrm{F}}$ \\
\hline LA & $45.2(8.9)$ & $44.3(8.3)$ & $0.613^{\mathrm{F}}$ \\
\hline $\mathrm{AO}$ & $32.8(4.9)$ & $31.9(5.8)$ & $0.484^{\mathrm{F}}$ \\
\hline RVD & $38.4(12.1)$ & $37.2(12.3)$ & $0.666^{\mathrm{F}}$ \\
\hline LVD & $45.9(8.7)$ & $47.2(8)$ & $0.504^{\mathrm{F}}$ \\
\hline LVS & $28.9(9.4)$ & $32.2(8.4)$ & $0.088^{\mathrm{F}}$ \\
\hline Fractional shortening & $38.2(11.1)$ & 33.7 (13) & $0.110^{\mathrm{F}}$ \\
\hline IVS & $12.8(2.7)$ & $12.3(2.7)$ & $0.383^{\mathrm{L}}$ \\
\hline EA ratio & $1.0(0.6)$ & $1.4(0.7)$ & $0.011^{\mathrm{L}}$ \\
\hline LV mass & 335.8 (138.9) & 273.8 (106.5) & $0.018^{\mathrm{L}}$ \\
\hline
\end{tabular}

$\mathrm{F}=P$-value for global test of any differences between BNP categories from ANOVA F-test. $\mathrm{L}=P$-value from ANOVA F-test, data $\log$ transformed prior to analysis. $\mathrm{C}=P$-value from chi-square test. $\mathrm{K}=P$ value from Kruskal-Wallis test.

Abbreviations and units same as Table 1.

vated BNP included LV mass, dimensions and ejection fraction, as well as gender, a diagnosis of diabetes, or history of ischemic heart disease.

Marked elevations of BNP are frequently seen in systolic heart failure and generally reflect increased wall stress due to left ventricular enlargement without compensatory wall thickening ${ }^{[11]}$. In our cohort, patients with $\mathrm{BNP}>1,000 \mathrm{pg} / \mathrm{mL}$ did not have evidence of increased wall stress, as their left ventricular end diastolic dimensions, wall thickness and left ventricular mass were similar to the other groups. These findings parallel those of Niizuma et al. ${ }^{[11]}$, who found no correlation between BNP concentrations and left ventricular end-diastolic wall stress in patients with HFpEF.

In contrast to patients with marked elevations of BNP, a recent study identified patients with a clinical diagnosis of HFpEF and normal BNP $\leq 100 \mathrm{pg} / \mathrm{mL}$ in $29 \%$ of outpatients ${ }^{[12]}$. Those patients were character- ized as younger women with obesity, but less frequently with CKD. In our study of inpatients with a BNP $\leq$ $100 \mathrm{pg} / \mathrm{mL}$ and a clinical diagnosis of $\operatorname{HFpEF}(\mathrm{n}=$ 54 , prevalence of $13 \%$ ), these patients, in comparison to those with a $\mathrm{BNP}>1,000 \mathrm{pg} / \mathrm{mL}$, were younger, had a lower prevalence of CKD IV (8\%) and CKD V $(5 \%)$, and less frequent use of anti-hypertensive medications. In contrast to the prior study, there was no difference in gender distribution. While the previously reported greater prevalence of normal BNP in outpatients likely represents the less severe disease found in the outpatient populations compared to those hospitalized with $\mathrm{HFpEF}$, these two studies indicate that there are common factors, primarily younger age and a low prevalence of significant $\mathrm{CKD}$, in patients with $\mathrm{HFpEF}$ and $\mathrm{BNP} \leq$ $100 \mathrm{pg} / \mathrm{mL}$.

\section{The role of CKD}

Elevated BNP in patients with chronic kidney disease may reflect the extent of cardiac dysfunction or, alternatively, altered metabolism of BNP in renal failure. While the relationship of BNP to left ventricular end-diastolic wall stress is significant in patients with end-stage renal disease and systolic dysfunction, that relationship was not found by Nizuma et al. in patients with preserved systolic function ${ }^{[2]}$. In that study, BNP was significantly elevated in those patients with endstage renal disease (GFR $<15 \mathrm{~mL} /$ minute $/ 1.73 \mathrm{~m}^{2}$ ), $93 \%$ of whom were on hemodialysis. However, BNP was not significantly elevated in patients with HFpEF who had CKD I-IV compared to those with normal renal function, suggesting that markedly elevated BNP is seen primarily in those with CKD V, generally on dialysis.

The clearance of BNP is two-fold. BNP binds to natriuretic peptide receptor $\mathrm{C}$ which is responsible for its clearance via receptor-mediated endocytosis and lysosomal degradation ${ }^{[13]}$, with subsequent removal of the peptide by the kidneys. BNP is also enzymatically degraded to an inactive form by neutral endopeptidase ${ }^{[14]}$. Thus, both reduced renal function and reduced activity of neutral endopeptidase can result in higher serum levels of BNP.

In the current study, there was a statistically significant inverse relationship with BNP and eGFR (Fig. 1). Furthermore, $17.5 \%$ of patients with a BNP > $1,000 \mathrm{ng} / \mathrm{mL}$ had a history of ESRD or were on hemodialysis, compared to $0,0.9$, and $5.7 \%$ in patients with BNP $\leq 100,100-400$, and 400-1,000, respectively. Thus, these data confirm the importance of severely impaired renal function in resultant levels of BNP $>$ $1,000 \mathrm{pg} / \mathrm{mL}$. 
Approximately one-fifth (21\%) of the patients with markedly elevated $\mathrm{BNP}>1,000 \mathrm{pg} / \mathrm{mL}$ had normal renal function (defined as eGFR $>60 \mathrm{~mL} /$ minute $/ \mathrm{m}^{2}$ ). Thus, while renal dysfunction contributes to elevated serum levels of BNP, it is not a requirement. Those patients without CKD appeared to be different than those with CKD, tending to have lower mean BNP values, and a lower prevalence of anti-hypertensive medications. There was a suggestion of lower systolic function in this cohort. While there were no differences between these groups in LV mass, these data suggest those patients with elevated BNP but without CKD may represent an elderly population with a lower burden of chronic hypertensive and renal disease, yet have elevated wall stress due to mildly impaired systolic function in addition to diastolic dysfunction ${ }^{[15]}$. Although speculative, another possible explanation for their markedly elevated BNP in the absence of CKD is reduced activity of neutral endopeptidase, as seen in patients with aging ${ }^{[16]}$.

The prognostic implications of markedly elevated BNP were paradoxical, as BNP $>100 \mathrm{pg} / \mathrm{mL}$ did not predict either subsequent death or myocardial infarction, yet patients with a relatively lower BNP during index hospitalization had a higher rate of subsequent hospitalization for heart failure. This may reflect the higher LV mass in patients with preserved renal function yet BNP $>1,000 \mathrm{pg} / \mathrm{mL}$ (Table 2). While this latter observation needs further study, these data suggest that, in contrast to systolic heart failure (HFrEF), the level of BNP in HFpEF does not seem to provide prognostic information regarding death or $\mathrm{MI}^{[3]}$.

\section{Limitations}

A strength of this study was the large number of patients included in a study of HFpEF. However, as a retrospective study from one institution, this data set was limited to observing differences in patients characterized by a clinical diagnosis of HFpEF in the inpatient setting. Thus, these data may not directly apply to outpatients. The diagnosis was established by the treating physician and, as a clinical diagnosis, it is possible that criteria for the diagnosis were not consistent across patients. Also, there are some data that were not obtained from the studies. For example, left atrial volume index was not consistently measured and was therefore not included in the echocardiographic parameters ${ }^{[9]}$.

In conclusion, marked elevations of $\mathrm{BNP}(>1,000 \mathrm{pg} /$ $\mathrm{mL}$ ) are common in patients with a clinical diagnosis of HFpEF, comprising $28 \%$ of the patients in this sample, and primarily characterized by severe CKD and hypertension. CKD is not obligatory, as approximately onefifth of patients with BNP $>1,000 \mathrm{pg} / \mathrm{mL}$ had normal renal function; these patients tended to be older with lower ejection fractions. In contrast to systolic heart failure, markedly elevated BNP does not confer a negative prognosis, perhaps because of confounding disease processes.

\section{Acknowledgements}

The project described was supported by the National Center for Advancing Translational Sciences (NCATS), National Institutes of Health (NIH), through grant \#ULl TR000002

\section{References}

[1] Wood P, Piran S, Liu PP. Diastolic heart failure: progress, treatment challenges, and prevention. Can J Cardiol 2011;27:302-10.

[2] Niizuma S, Iwanaga Y, Yahata T, Tamaki Y, Goto Y, Nakahama H, et al. Impact of left ventricular end-diastolic wall stress on plasma B-type natriuretic peptide in heart failure with chronic kidney disease and end-stage renal disease. Clin Chem 2009;55:1347-53.

[3] Maeda K, Tsutamoto T, Wada A, et al. High levels of plasma brain natriuretic peptide and interleukin-6 after optimized treatment for heart failure are independent risk factors for morbidity and mortality in patients with congestive heart failure. J Am Coll Cardiol 2000;36:158793.

[4] van Veldhuisen DJ, Linssen GC, Jaarsma T, van Gilst WH, Hoes AW, Tijssen JG, et al. B-type natriuretic peptide and prognosis in heart failure patients with preserved and reduced ejection fraction. J Am Coll Cardiol 2013; 14:1498-506.

[5] Lang RM, Bierig M, Devereux RB, Flachskampf FA, Foster E, Pellikka PA, et al. Recommendations for chamber quantification: a report from the American Society of Echocardiography's Guidelines and Standards Committee and the Chamber Quantification Writing Group, developed in conjunction with the European Association of Echocardiography, a branch of the European Society of Cardiology. Journal of the American Society of Echocardiography: official publication of the American Society of Echocardiography 2005;18:1440-63.

[6] Agresti A. An introduction to categorical data analysis. New York, N.Y.: John Wiley \& Sons, 2007.

[7] O’Meara E, Chong KS, Gardner RS, Jardine AG, Neilly JB, McDonagh TA. The Modification of Diet in Renal Disease (MDRD) equations provide valid estimations of glomerular filtration rates in patients with advanced heart failure. Eur J Heart Fail 2006;8:63-7.

[8] Baggish AL, Lloyd-Jones DM, Blatt J, Richards AM, Lainchbury J, O’Donoghue M, et al. A clinical and biochemical score for mortality prediction in patients with acute dyspnoea: derivation, validation and incorporation into a bedside programme. Heart 2008;94:1032-7.

[9] Iwanaga Y, Nishi I, Furuichi S, Noguchi T, Sase K, Kihara Y, et al. B-type natriuretic peptide strongly reflects 
diastolic wall stress in patients with chronic heart failure: comparison between systolic and diastolic heart failure. $J$ Am Coll Cardiol 2006;47:742-8.

[10] Jaubert MP, Armero S, Bonello L, Nicoud A, Sbragia P, Paganelli F, et al. Predictors of B-type natriuretic peptide and left atrial volume index in patients with preserved left ventricular systolic function: an echocardiographiccatheterization study. Arch Cardiovasc Dis 2010;103:3-9.

[11] Maisel AS, McCord J, Nowak RM, Hollander JE, Wu $\mathrm{AH}$, Duc $\mathrm{P}$, et al. Bedside B-Type natriuretic peptide in the emergency diagnosis of heart failure with reduced or preserved ejection fraction. Results from the Breathing Not Properly Multinational Study. J Am Coll Cardiol 2003:41:2010-7.

[12] Iwanaga Y, Kihara Y, Niizuma S, Noguchi T, Nonogi H, Kita T, et al. BNP in overweight and obese patients with heart failure: an analysis based on the BNP-LV diastolic wall stress relationship. J Card Fail 2007;13:663-7.
[13] Anjan VY, Loftus TM, Burke MA, Akhter N, Fonarow GC, Gheorghiade M, et al. Prevalence, clinical phenotype, and outcomes associated with normal B-type natriuretic Peptide levels in heart failure with preserved ejection fraction. Am J Cardiol 2012;110:870-6.

[14] Daniels LB, Maisel AS. Natriuretic peptides. J Am Coll Cardiol 2007;50:2357-68.

[15] Vanderheyden M, Bartunek J, Goethals M. Brain and other natriuretic peptides: molecular aspects. Eur $J$ Heart Fail 2004;6:261-8.

[16] Morris DA, Boldt LH, Eichstä dt H, Ozcelik C, Haverkamp W. Myocardial systolic and diastolic performance derived by 2-dimensional speckle tracking echocardiography in heart failure with normal left ventricular ejection fraction. Circ Heart Fail 2012;5:610-20.

[17] Reckelhoff JF, Baylis C. Proximal tubular metalloprotease activity is decreased in the senescent rat kidney. Life Sci 1992;50:959-63. 